\title{
Micturition symptoms in frontal tumours
}

\author{
R. S. MAURICE-WILLIAMS ${ }^{1}$
}

From the Neurosurgical Unit of Guy's, Maudsley, and King's College Hospitals, London

SYNOPSIS In a series of 50 consecutive tumours involving the frontal lobes, seven cases $(14 \%)$ were found who exhibited the syndrome, first described by Andrew and Nathan in 1964, of frequency of micturition, urgency, and incontinence causing distress to the patient. This suggests that this syndrome may occur rather more commonly than those authors had indicated. In 100 consecutive intracranial tumours, no instances were found with non-frontal tumours, indicating that the syndrome may be of localizing value.

It is well known that incontinence of urine can occur in frontal tumours as part of a frontal lobe syndrome of indifference, disinhibition, and selfneglect. What is less widely recognized is that frontal tumours can also give rise to a syndrome of frequency of micturition, urgency, and incontinence in the absence of any signs of intellectual or moral deterioration. On occasion, a patient may present himself with these symptoms and be treated for a while for a supposed disorder of the urinary tract. This syndrome was first delineated by Andrew and Nathan (1964) who reported cases occurring with a variety of frontal lobe lesions, including nine tumours, collected over a period of 24 years.

Seven further cases due to tumours are reported here. These seven cases occurred among 50 consecutive tumours involving the frontal lobes $(14 \%)$, suggesting that the syndrome may be commoner than Andrew and Nathan's paper would indicate. No instances other than with frontal tumours could be found in a series of 100 consecutive intracranial tumours, confirming that the syndrome may be of value in localizing a tumour to the frontal region.

\section{CASE 1}

(GM. 9561) A 68 year old retired nurse consulted her doctor because of urinary frequency and urgency. On one or two occasions she had had nocturnal incontinence. When these symptoms failed to

1 Present address: Department of Neurosurgery, St. Bartholomew's Hospital, London E.C.1. respond to treatment she was referred to a urologist who could find no abnormality on pyelography or cystoscopy. It was now six months since the onset of her symptoms. While in hospital for the cystoscopy, it was noticed that she had some slight weakness of the left arm, but as she had been known to be hypertensive for some years, this was attributed to a small 'stroke'. Over the next few months she began to have headaches and to drag her left foot. When she began to become forgetful, irritable, and careless of her appearance she was readmitted to hospital. By now, one year from the time of her original urinary symptoms, she was obviously dementing, and had a marked left hemiparesis. A spherical meningioma $6 \mathrm{~cm}$ in diameter was removed from the outer half of her right sphenoidal ridge. The tumour had buried itself in the right frontal lobe. Postoperatively she made a complete recovery and returned home. After the first week postoperatively she had good control of micturition, without either frequency or urgency.

\section{CASE 2}

(GM. 9434) A 48 year old draughtsman was admitted to hospital in July 1972. He was known to have a right medial frontal oligodendroglioma, adjacent to the genu of the corpus callosum, as this had been producing general and focal fits for 10 years, and five years previously had been confirmed by biopsy. In January 1972 he began to have headaches, and noticed that he was becoming slowed up and that his memory was failing. He was troubled by sudden attacks of urinary urgency and often wet himself before he could reach the lavatory. He was also incontinent of urine at night. He consulted his doctor because of these urinary symptoms and because of failing visual acuity. The fits had involved no special 
disturbance of micturition, though he had been incontinent of urine during the course of some grand mal attacks. He could recall no sensation of urgency during the aura. On examination he was alert, well orientated, and sensible, but easily distractable. $\mathrm{He}$ had no dysphasia but had a minimal left faciobrachial weakness, and had bilateral papilloedema, with paralysis of conjugate gaze to the left.

He made a good recovery after subtotal removal of his tumour. For the first few postoperative days he was incontinent of urine, but four weeks later he had no urinary symptoms whatsoever, although his mental state had not greatly improved by that time.

\section{CASE 3}

(GM. 6824) A 40 year old research chemist developed morning headaches with nausea. After a month he developed attacks of extreme urinary urgency. He felt that his bladder was about to void but often could not control it. These attacks occurred once or twice a day, gradually increased in frequency, and on a few occasions he wet himself at night. On occasions he had had nocturnal faecal incontinence. During this time his mental state and behaviour remained normal but two months after the onset of his urinary symptoms he became drowsy and was admitted to hospital. A malignant glioma was partially removed from the posteromedial part of his right frontal lobe. It appeared to be invading the anterior end of the corpus callosum. Six days after the operation, in which the right frontal lobe had also been removed, he was free of all urinary symptoms, and they have not recurred after 26 months.

\section{CASE 4}

(GM. 8652) A 51 year old secretary was admitted to hospital with a two-week history of urinary frequency with extreme urgency, nocturia of three times a night, and a tendency to lose the track of a conversation. She also complained that she tended to veer to the right when walking. On admission she was found to be fully orientated and sensible but showed a slight nominal dysphasia and tended to fall to the right on Romberg's test. Two days later a mild right-sided weakness became evident, and over the next week she became drowsy and grossly dysphasic. Carotid angiography showed a left posterior frontal intrinsic malignant tumour, which biopsy confirmed as a malignant glioma. She rapidly deteriorated and died two weeks postoperatively. At postmortem examination the tumour was found to be $5 \mathrm{~cm}$ across and lying in the upper part of the left posterior frontal lobe.

\section{CASE 5}

(GM. 9381) A 60 year old painter had been aggressive, argumentative, and sometimes confused in conversation for six months. For six weeks he had had frontal headaches and on several occasions voided urine before he could reach the lavatory. A week before admission to hospital he became confused and then drowsy. On arrival he was stuporose, with papilloedema and a slight left lower facial weakness. The right frontal lobe was resected to- 0 gether with part of a $6 \mathrm{~cm}$ diameter necrotic glioma which occupied its posterior half. After operation, he became fully orientated and alert with no disturbance of micturition for a period of four weeks. His condition then gradually deteriorated, and he became apathetic, dishevelled, and doubly incontinent, being indifferent to this incontinence, in contrast with the anxiety he had shown about his previous attacks of precipitate urinary incontinence.

\section{CASE 6}

(GM. 9273) A 52 year old housewife suddenly. found that she was liable to wet herself at all times of day without warning. A week later she developed b\$ temporal headaches and felt slowed up and ing decisive. She found it difficult to concentrate wheg $\rightarrow$ driving. One month after the onset of her symptoms she was admitted to hospital. She was found to apathetic and inattentive, with bilateral papilloedem $\vec{v}$ but no focal neurological signs. A carotid angiogra showed a large avascular mass at the right frontat pole and at operation a glioblastoma multiforme on the medial side of the right frontal pole was subtotally removed. When discharged from hospital two weeks after operation she had returned to her normal mental state, and had good control over micturition, but with nocturnal frequency. Six months later she was leading a normal life with no urinary symptoms at all.

\section{CASE 7}

(GM. 9620) A 57 year old engineer visited his family doctor for the first time, complaining of urinary frequency, frontal headaches, and difficulty in concentrating at work for two weeks. His doctor could find no abnormality on examination, but over the next week he became intermittently drowsy and confused, and was admitted to hospital. By that time $\frac{7}{0}$ he was stuporose and had a mild left faciobrachial weakness. A carotid angiogram revealed an avascu- N lar mass at the right frontal pole, with considerable $N$ shift of the midline vessels to the left. A burr-hole biopsy confirmed a malignant glioma. Treatment with large doses of betamethasone caused all his 
symptoms and signs to clear including his urinary frequency. Later, a frontal lobectomy with subtotal removal of the tumour was performed without relapse of his micturition symptoms.

\section{COMMENT}

These seven cases were seen in a series of 50 consecutive frontal tumours which passed through a single surgical firm at the Guy'sMaudsley Neurosurgical Unit over a period of 29 months, from May 1969 to October 1972. The first 20 cases of this series were reviewed retrospectively, which permits the objection that insufficient detail was obtainable. However, details of sphincter function are routinely asked for in the clerking notes of this unit, and omission of these details would, if anything, have led to an underestimate of the incidence of this syndrome. Four further cases had a history of urinary incontinence which appeared to be related to a general intellectual deterioration. These four cases are excluded.

Of the seven cases, four were men, three women, their ages ranging from 40 to 68 years. Six of the tumours were on the right side, one on the left. They comprised five malignant gliomas, one meningioma, and one oligodendroglioma, as compared with the total series of 50 tumours, which comprised 31 malignant gliomas, 12 meningiomas, five metastases, and two oligodendrogliomas.

When micturition symptoms began, four patients had symptoms of raised intracranial pressure, and four showed some evidence of impairment of intellectual function, although none was apathetic or indifferent to his micturition symptoms. Only one patient had fits at any time, and these did not appear to be related in any way to his disturbance of micturition. Only one patient had developed any definite lateralizing features (a tendency to veer to one side) at the time that the micturition symptoms began. A single patient had disturbed defaecation in the form of nocturnal faecal incontinence. Of the micturition symptoms, urgency was complained of in five cases, daytime incontinence in four, nocturnal incontinence in three, daytime frequency in three, and nocturia in one.

In six cases the tumour was at least partially removed. In five of these the micturition symptoms remitted postoperatively, and in one the patient retains normal micturition function after 26 months. In the sixth case, preoperative steroid therapy had already led to a remission.

When the cases described here had been collected, the records were reviewed of 100 consecutive intracranial tumours, including the latter 30 of the frontal lobe cases, to see if any instances could be found of this syndrome in tumours other than frontal ones. None could be found. Of the 100 tumours, micturition symptoms were mentioned in six, all of them frontal tumours. Of these, three were cases of the syndrome described here, and in the other three the symptoms were of incontinence of urine as part of the better-known 'frontal lobe syndrome'. (The locations of the 100 tumours were as follows: frontal, 30; temporal, 15; parietal, seven; occipital, two; posterior fossa, 22; deep midline structures, 17; sella or parasellar, seven.)

\section{DISCUSSION}

The syndrome in which a lesion of the frontal lobe of the brain is accompanied by frequency and urgency of micturition, with occasional incontinence, causing distress to the patient, was first delineated by Andrew and Nathan (1964). Over a period of 24 years these authors had collected instances of the syndrome caused by a variety of frontal lesions: tumours, haematomas, penetrating injuries, and leucotomies. In a minority of their cases there was retention of urine, and in a few there were disturbances of defaecation, either frequency with incontinence, or constipation. By correlation of data, they came to the conclusion that there exists a centre involved in the control of micturition and defaecation which is located in the superomedial part of the frontal lobes, in the region of the anterior cingulate gyrus, the adjacent part of the superior frontal gyrus, and the underlying white matter, whose fibres are running to and from the genu of the corpus callosum.

Andrew and Nathan described nine cases in which the syndrome had been associated with a frontal tumour. Their cases differed in several respects from those reported here. Five of their nine cases were meningiomas, as opposed to only one of seven here. This, however, may merely be a reflection of the fact that unusual clinical features are brought to notice more easily in a 
slowly evolving tumour. None of the cases reported here had retention of urine, as compared with four in the previous series, and only one had a defaecation disturbance (nocturnal incontinence) as compared with three (one each of frequency, incontinence, and constipation). However the most striking difference between the two groups relates to seizures. In this series, only one patient had epileptic fits, consisting of either typical grand mal, sometimes with incontinence of urine, or of focal motor seizures in which no micturition features occurred. On the other hand, eight of nine patients in Andrew and Nathan's series had seizures, and in three of these the seizure was preceded by a strong desire to micturate. Altogether, four of their patients were incontinent of urine during fits. In some of their patients there appeared to be a disturbance of the sensations concerned with micturition. In six of their tumour cases there was an absence of the sensation of bladder fullness, and in four the sensation of the imminence of micturition appeared absent. No such disturbances of sensation are to be found among the patients in this series. In several of their patients, notably those subjected to leucotomies of the Lyerly-Poppen type, cystometrograms were made and these gave a clue to the nature of the underlying mechanism. When fluid was run into the bladder it was found that detrusor contractions occurred at a low level of bladder distension, the contractions often being impossible to inhibit, so that incontinence occurred. Sensations of bladder fullness were not present at degrees of bladder distension which would normally cause them. The result of these changes was that the bladder had a small functional capacity. There seemed to be a loss of higher control of the micturition reflex, which came into operation at a lower level of bladder distension than normal. The patient thus suffers no loss of concern about micturition, but finds that at perhaps frequent intervals he is overcome by an intense sensation of impending voiding of urine, which he may or may not be able to control in time. Andrew and Nathan found that in some of their most severe cases, even the sensation of imminence of voiding appeared to be diminished. In some respects the symptoms are similar to those which may occur early in spinal cord lesions, whether intrinsic or compressive. In cats when the cerebral cortex is removed, the volume of bladder fluid required to initiate micturition is lowered (Langworthy and Kolb, 1933).

In connection with the apparent dissociation which Andrew and Nathan noted of the sensa- of tion of bladder fullness and that of imminence of micturition, it is of interest to note that even at spinal cord level these sensations are conveyed in anatomically different sites, the spinothalamic $\vec{\Rightarrow}$ tracts and the dorsal columns respectively. The evidence for this is that a bilateral cordotomy will abolish the one sensation but not the other $\frac{\bar{m}}{\bar{m}}$ (Nathan and Smith, 1951). The sensation of $\overrightarrow{\mathbb{D}}$ urgency probably arises from the posterior urethra, that of fullness from the bladder itself o (Hofman, 1969). That the two sensations may become separated with frontal lesions suggests that their highest terminations as well as their ascending pathways may be separately arranged.

It is odd that the syndrome may be relieved by excision of both the causative lesion and the area of brain it involves. In five of the cases reported here, resection relieved the micturitio $\mathbb{\omega}_{\vec{S}}$ symptoms for up to 26 months. This sugges that the phenomenon may be a positive rathe than a negative one, the lesion activating somg system rather than releasing one from controt The possibility of some sort of epileptic phen $\overrightarrow{0}$ menon suggests itself, but against it is the fact that of these seven cases only one had any seizures, and these appeared not to be related to his micturition disturbances.

Bilateral excision of the frontal lobes as far back as the sphenoidal ridges and the main trunks of the anterior cerebral arteries does not give rise to other than transient micturition symptoms. One such case, in which bilaterally damaged frontal lobes causing fits and behaviour disturbances were excised, is reported in detail by Hebb and Penfield (1940). No postoperative disturbance of micturition ensued. In a report on 14 bifrontal lobectomies performed for various psychiatric disorders (Peyton et al., 1948) incontinence of urine postoperatively was recorded in 10. However, in no case did it continue for more than one month, and in seven of the 10 it appeared to be related to confusion or apathy.

The incidence $(14 \%)$ of the syndrome among frontal tumours reported here suggests that it may be more common than might be inferred from the paper of Andrew and Nathan. This is 
supported by the fact that Gautier-Smith (1970) in his monograph on falcine and parasagittal meningiomas found 17 cases where this syndrome occurred among 194 anteriorly and centrally placed tumours. If it is assumed that many of the 194 tumours were retrofrontal, the incidence among those involving the frontal lobes was probably approximately the same as that reported here. The syndrome appears not to occur with non-frontal tumours and so may be of localizing value, particularly as it seems that it may be present before other focal neurological features, as in six of the seven cases described here. It is possible that many cases are overlooked as, by the time the patient is admitted to hospital, progression of the tumour has led to intellectual deterioration or clouding of consciousness which obscures the patient's original distress at his micturition symptoms, these symptoms being attributed to a 'frontal lobe syndrome' of apathy and disinhibition. The latter might in fact be less common than the syndrome reported here. In comparison with the seven cases of this syndrome among 50 frontal tumours, there were only four cases of incontinence of urine related to apathy and indifference. In the meningioma series of Gautier-Smith (1970), the corresponding ratio was 17 to 11 .

What is remarkable is the fact that large and extensive frontal tumours may occur without micturition symptoms, even if Andrew and Nathan's postulated critical area is directly affected. It is possible that there is an individual variation in susceptibility to these symptoms and that most patients have an asymptomatic alteration of micturition function. This possibility is supported by the fact that Andrew and Nathan found that some leucotomy patients who did not complain of micturition symptoms postoperatively nevertheless were found on cystometrography to have changed from their preoperative state.

Andrew and Nathan derive their most convincing evidence for localization within the frontal lobes of a special area concerned with the control of micturition and defaecation from their leucotomy cases. They comment that the information derived from their tumour cases does not permit of any precise localization. It would certainly seem that the presence of the micturition syndrome will permit localization of a tumour only to the frontal region and not to within a particular part of the frontal lobes. Case 1 here, and a similar case of Andrew and Nathan, both large sphenoidal wing meningiomas, certainly did not directly involve the postulated critical area, and if such a critical zone exists they can have affected it only by indirect distortion. However, evidence is provided by tumour cases that the area involved with micturition control lies forward of the motor strip and Broca's area. Except possibly in case 4, all the cases reported here seem to have developed micturition onsets before the onset of either dysphasia or focal weakness.

The apparent preponderance of right-sided tumours (six of seven here, 12 of 16 if both series are taken together) may not be significant. It is possible that the apparent paucity of dominant hemisphere cases has resulted from the subsequent onset of dysphasia obscuring the exact nature of the earlier symptoms due to inability to recount them. Andrew and Nathan have pointed out that lesions in their postulated area would involve fibres travelling to and from the genu of the corpus callosum and hence could be placed in either hemisphere.

Disturbances of defaecation appear to be much less common than those of micturition. They occurred in only one case here (case 3, who had nocturnal faecal incontinence) and in three of Andrew and Nathan's tumour cases. The latter suggest that this may be because faeces is generally not fluid and because the rectum fills less rapidly than the bladder. On the other hand, the evidence for a specific disturbance of defaecation is not as clear-cut as for micturition, and most of those cases where such disturbances have apparently occurred are explicable on other grounds-for example, constipation from analgesics, or nocturnal incontinence from occult seizures. None of Andrew and Nathan's patients subjected to the Lyerly-Poppen type of leucotomy showed disturbance of defaecation, although this procedure clearly disturbed micturition.

I wish to thank Mr. J. J. Maccabe for permission to report on cases treated under his care, and Professor C. D. Marsden, for his helpful advice and criticism.

\section{REFERENCES}

Andrew, J., and Nathan, P. W. (1964). Lesions of the anterior frontal lobes and disturbances of micturition and defaecation. Brain, 87, 233-262. 
Gautier-Smith, P. C. (1970). Parasagittal and Falx Meningiomas. Butterworths: London.

Hebb, D. O., and Penfield, W. (1940). Human behavior after extensive bilateral removal from the frontal lobes. Archives of Neurology and Psychiatry, 44, 421-438.

Hofman, P. (1969). Bladder and sphincter functions and their disorders. In Handbook of Clinical Neurology, Vol. 1, pp. 356-403. Edited by P. J. Vinken and G. W. Bruyn. NorthHolland: Amsterdam.

Langworthy, O. R., and Kolb, L. C. (1933). The encephalic control of tone in the musculature of the urinary bladder Brain, 56, 371-382.

Nathan, P. W., and Smith, M. C. (1951). The centripetal pathway from the bladder and urethra within the spinal cord. Journal of Neurology, Neurosurgery, and Psychiatry, 14, 262-280.

Peyton, W. T., Noran, H. H., and Miller, E. W. (1948). Prefrontal lobectomy. American Journal of Psychiatry, 104, 513-523. 